\title{
Hardness, polymerization depth, and internal adaptation of Class II silorane composite restorations as a function of polymerization protocol
}

\author{
Janaina Bechtold ${ }^{1}$ \\ Priscila Jaques dos Santos ${ }^{1}$ \\ Andrea Anido-Anido ${ }^{2}$ \\ Vinícius Di Hipólito² \\ Roberta Caroline Bruschi Alonso ${ }^{2}$ \\ Paulo Henrique Perlatti D'Alpino
}

\begin{abstract}
Objectives: To evaluate the influence of various photoactivation techniques on the internal gap, Knoop-hardness, and polymerization depth of silorane- and methacrylate-based composites in Class II restorations.
\end{abstract}

Methods: Preparations were made in third molars ( $n=10$ ), according to composites (Filtek P60: methacrylate; Filtek P90: silorane) and photoactivation techniques (OC: occlusal photoactivation (control); OBL: occlusal+buccal+lingual photoactivation; and BLO: buccal+lingual+occlusal photoactivation (transdental)). Composites were inserted in two increments, both individually photoactivated for 20 s. After $24 \mathrm{~h}$, specimens were sectioned and the ratio of internal gaps to interface length (\%) recorded. Hardness was tested across the transversal section of restorations (1-4 $\mathrm{mm}$ below the surface).

Results: Silorane restorations showed significantly lower gaps compared with methacrylate, regardless of polymerization technique $(P<.05)$. Supplementary energy dose in OBL and BLO protocols caused significant increase in gaps in silorane restorations $(P<.05)$. For methacrylate restorations, $\mathrm{OBL}$ activation caused significantly higher gap formation $(\mathrm{P}<.05)$. Significantly lower hardness values were seen for silorane than for methacrylate composites $(\mathrm{P}<.05)$, regardless of depth and photoactivation. Significantly higher hardness values were seen in BLO activation for methacrylate restorations compared with control $(\mathrm{P}<.05)$; for silorane, no differences were observed. Significantly higher hardness values were observed at 1 and $3 \mathrm{~mm}$ compared to 2 and $4 \mathrm{~mm}$ for both composites.

Conclusions: Internal gaps and hardness are affected by composite type and photoactivation. Despite the reduced values, hardness of silorane is not influenced by photoactivation or by depth. Internal gaps are dependent on the energy dose for both composites, with silorane showing lower internal gaps. (Eur J Dent 2012;6:133-140)

Key words: Dental composites; Knoop hardness; polymerization; internal adaptation

1 Anhanguera-Uniban University, São Paulo, SP, BRASIL

2 Biomaterials Research Group, Anhanguera-Uniban University, São Paulo, SP, BRASIL
Corresponding author: Dr. Paulo Henrique Perlatti D’Alpino Universidade Anhanguera-Uniban Rua Maria Cândida, 1.813, Bloco G, 6. ${ }^{\circ}$ andar, São Paulo - SP, CEP: 02071-013, BRASIL Tel: +55 1129679058

Email: paulodalpinodyahoo.com 


\section{INTRODUCTION}

The main problem related to composite restorations is the polymerization shrinkage that creates contraction forces that lead to stress accumulation at the preparation walls; as a consequence, gap formation and subsequent microleakage can occur. ${ }^{1,2}$ Several approaches have been proposed to reduce the resulting shrinkage stress such as controlling cavity configuration (C-factor), ${ }^{3}$ modulating light intensity with different polymerization techniques, ${ }^{4}$ using different cavity filling methods, ${ }^{5}$ and also applying stress absorbing intermediate layers. ${ }^{6,7}$ In addition, new formulations of composites have been developed by increasing the volume of inorganic particles, increasing the monomer molecular weight, or modifying the chemical structure of certain monomers and/or replacing them..$^{8,9}$

Recently, a silorane-based composite was introduced containing cationic ring-opening monomers, a compensating mechanism for shrinkage stress occurring during polymerization. ${ }^{10}$ This new monomer system, called silorane, was obtained from the reaction of oxirane and siloxane molecules. ${ }^{10}$ It has been claimed that the novel resin combines the two key advantages of the individual components: low polymerization shrinkage due to the ring-opening oxirane monomer and increased hydrophobicity due to the presence of the siloxane species. As a result of these particular characteristics, the silorane-based composite revealed decreased water sorption, solubility, and associated diffusion coefficient compared with these qualities when methacrylate-based composites were tested. ${ }^{11}$ In a previous study, ${ }^{11}$ it was found that the cusp deflection caused by polymerization shrinkage was significantly lower when extracted teeth were restored with an experimental silorane material in comparison to that seen when a methacrylate-based composite was applied. In addition, in another study, ${ }^{12}$ it was found that no microleakage occurred when Class II MOD preparations were restored with a silorane-based composite. On the other hand, a clinical study ${ }^{13}$ revealed that the excellent results exhibited by silorane composite restorations in laboratory tests were not clinically validated. In that study, the marginal adaptation of direct Class II silorane composite restorations was evaluated immediately and after one year. Results were compared with those of methacrylate composite restorations. At the follow-up evaluation, marginal adaptation in- dicated better performance of methacrylate-based composite restorations.

A wide variety of light sources is currently available, with increased power density and different spectral irradiance, with inherent characteristics and claimed advantages. ${ }^{14,15}$ On the other hand, the best irradiation procedure for polymerizing composites has not been determined yet.4,16 These parameters are of particular interest since, in practice, they are under control of the clinician. ${ }^{17}$ Application of initial low-intensity polymerization techniques has been proposed to reduce irradiance level to activate the material. ${ }^{18}$ An initial slow curing allows slow development of composite stiffness and favors composite flow, helping to reduce shrinkage stress. ${ }^{19}$ In one of these techniques, known as transdental technique, ${ }^{20}$ the light is irradiated through the dental tissues. In this case, there is a reduction of up to $70 \%$ in the power density that reaches the composite at the other side of the dental structure. ${ }^{21}$ One may argue that such method could modify the kinetics of polymerization as well as crosslink density, ${ }^{22}$ leading to different polymer structures, despite similar degrees of conversion. ${ }^{23}$ Crosslink density has been associated with increased physical properties and stability; ${ }^{23}$ thus, composites with poor mechanical properties would be obtained. ${ }^{24,25,26}$ Although transdental technique provided no benefits in terms of marginal adaptation, a significant increase in the mechanical properties of certain methacrylate-based composites was seen. ${ }^{27}$

This study evaluated the influence of different polymerization protocols on the composite microhardness and internal adaptation of Class II restorations filled with different posterior restorative systems la silorane-based restorative system and a methacrylate-based composite system). The following research hypotheses were tested for both restorative systems by using the methacrylatebased composite as a reference: (1) restorations filled with the silorane-based composite would present reduced gap formation; (2) the transdental polymerization technique would reduce gap formation without decreasing composite hardness.

\section{MATERIALS AND METHODS}

Specimen preparation

A total of 30 sound, recently extracted human third molars were scaled, cleaned with a slurry of 
pumice and water, and stored in a $0.1 \%$ thymol solution at room temperature to prevent bacterial growth for no more than a month. Teeth were obtained and used in accordance with a protocol approved by the local Ethical Committee (file number: COPI 2009334). The cusps were abraded using a wet-ground \#320-grit silicon carbide paper and then finished with \#600-grit. Standardized, Class II preparations at both mesial and distal surfaces of the teeth were made using \#245 carbide burs (Brasseler, Savannah, GA, USA) in a high-speed handpiece. Preparation dimensions were the following: bucco-lingual width $4.0 \mathrm{~mm}$; gengivo-occlusal width $5.0 \mathrm{~mm}$; axial wall $2.5 \mathrm{~mm}$ deep. The occlusal margins were located in enamel, and the gingival margin was located in dentin. Burs were replaced after three preparations. Preparation finishing was performed dry with the same bur at low speed. For each tooth, one preparation was restored using the methacrylate-based restorative system Filtek P60 (3M/ESPE, St Paul, MN, USA), and the other using the silorane-based restorative system composite Filtek P90 (3M/ESPE, St Paul, MN, USA). The characteristics of the composites as well as the selected dentin bonding adhesive systems are described in Table 1. All restorative materials were photoactivated using an LED light-curing unit (Bluephase, Ivoclar-Vivadent, Schaan, Liechtenstein) with a power density of $1,000 \mathrm{~mW} / \mathrm{cm}^{2}$.

The preparation to which the methacrylatebased composite was applied was previously etched for $15 \mathrm{~s}$ with a $35 \%$ phosphoric acid gel (Scotchbond Etchant, 3M/ESPE, St Paul, MN, USA) and then water rinsed for $20 \mathrm{~s}$. Absorbent paper tissue was used to remove excess water inside the preparation. A dentin bonding adhesive system (Adper Single Bond 2, 3M/ESPE, St Paul, MN, USA) was applied to all aspects of the preparation, following manufacturer's instructions. The preparations were previously etched for $15 \mathrm{~s}$ with $35 \%$ phosphoric acid gel (Scotchbond Etchant, 3M ESPE, St. Paul, MN, USAl and then water rinsed for $20 \mathrm{~s}$. Two layers of adhesive were applied to all aspects of the preparation and a gentle air blowing for $3 \mathrm{~s}$ was performed. Adhesive was then polymerized for $10 \mathrm{~s}$. In the opposite preparation receiving the silorane-based composite, a two-step, self-etching adhesive system (P90 System adhesive, 3M/ESPE, St Paul, MN, USAl was applied to all aspects of the preparation also following manufacturer's instructions. P90 Primer was then applied for $15 \mathrm{~s}$ with a microbrush, followed by gentle air dispersion and $10 \mathrm{~s}$ of light curing. P90 Bond was also applied with a microbrush, followed by gentle air dispersion and photoactivation for $10 \mathrm{~s}$.

After bonding procedures, a Mylar strip attached to a Tofflemire matrix retainer was fixed around the tooth. The specimen was then placed into a device containing two adjacent teeth to simulate proximal contact. Wood wedges were inserted in the proximal areas to provide proximal contact and contour with the adjacent tooth as well as to provide an adequate cervical adaptation. Both composites (shade A3) were inserted in two increments ( $2.5 \mathrm{~mm}$ thick), which were individually photoactivated. Specimens were randomly divided into three groups ( $n=10$ ),

Table 1. Description of the adhesives and composites used in the study.

\begin{tabular}{|c|c|c|}
\hline Product name & Ingredients & lot \# \\
\hline $\begin{array}{l}\text { Filtek P90 } \\
\text { (3M ESPE) }\end{array}$ & $\begin{array}{l}\text { 3,4-Epoxycyclohexylethylcyclopolymethylsiloxane, } \\
\text { bis-3,4-epoxycyclohexylethylphenylmethylsilane; } \\
\text { Silanized quartz; yttrium fluoride; } 76 \text { wt } \% \text {. }\end{array}$ & 9ER \\
\hline $\begin{array}{l}\text { Filtek P60 } \\
\text { (3M ESPE) }\end{array}$ & $\begin{array}{c}\text { Bis-GMA; Bis-EMA; UDMA; TEGDMA; } \\
\text { Silica nanofiller; } 83 w t \% .\end{array}$ & 9PG \\
\hline $\begin{array}{l}\text { P90 System Adhesive } \\
\text { (3M ESPE) }\end{array}$ & $\begin{array}{l}\text { Two-bottle self-etch adhesive system; } \\
\text { Primer: phosphorylated methacrylates, Vitrebond } \\
\text { copolymer, Bis-GMA, HEMA, water, ethanol, } \\
\text { silane-treated silica filler, initiators, stabilizers. } \\
\text { Bond: hydrophobic dimethacrylate, phosphorylated } \\
\text { methacrylates, TEGDMA, silane-treated silica filler, } \\
\text { initiators, stabilizers. }\end{array}$ & $\begin{array}{l}9 \mathrm{BL}(\mathrm{P}) \\
9 \mathrm{BH}(\mathrm{B})\end{array}$ \\
\hline $\begin{array}{l}\text { Adper Single Bond } 2 \\
\text { (3M ESPE) }\end{array}$ & $\begin{array}{l}\text { Etch-and-rinse, conventional adhesive system; } \\
\text { Bis-GMA; polyalkenoic acid co-polymer; dimethacrylates; } \\
\text { HEMA; photoinitiators; ethanol; water; nanofiller particles. }\end{array}$ & $9 \mathrm{WF}$ \\
\hline
\end{tabular}


according to the following photoactivation techniques:

1. OC: occlusal: occlusal irradiation for $20 \mathrm{~s}$;

2. OBL: $20 \mathrm{~s}$ occlusal, $20 \mathrm{~s}$ buccal, and $20 \mathrm{~s}$ lingual irradiation, sequentially;

3. BLO: transdental: $20 \mathrm{~s}$ buccal, $20 \mathrm{~s}$ lingual, and 20 s occlusal irradiation, sequentially.

For the occlusal photoactivation, the distal end of the light curing was positioned close to the occlusal surface of the restoration, and continuous irradiation at $1,000 \mathrm{~mW} / \mathrm{cm}^{2}$ was performed. When irradiation was performed through the dental structures, the distal end of the light curing was positioned buccally and lingually to the restoration, and a decrease to $160 \mathrm{~mW} / \mathrm{cm}^{2}$ power density was obtained. Considering these differences in power density, the energy dose calculated for OC was $20 \mathrm{~J}$ and for OBL and BLO was $26.4 \mathrm{~J}$.

Specimens were then stored at $37^{\circ} \mathrm{C}$ in physiologic saline solution for $24 \mathrm{~h}$. Restorations were then finished and polished using 8- and 16-blade carbide burs (ET4 and ET3F, Brasseler, Savannah, GA, USA, respectively) and disks (Sof-Lex, 3M ESPE, 3M/ESPE, St Paul, MN, USA) mounted in a slow-speed handpiece. The specimens were subsequently embedded in epoxy resin (Castin' Craft Clear Liquid Plastic, Environmental Technology Inc., Fields Landing, CA, USA), allowing each tooth to be mounted and sectioned using a water-cooled rotating diamond blade (Isomet Low Speed Saw, Buehler Ltd., Evanston, IL, USA). Each restoration was mesio-distally sectioned. Both resulting surfaces were examined, but results of the two sections were taken as a single data. After $24 \mathrm{~h}$, each specimen was wet-polished with 600-, 1,200-, and

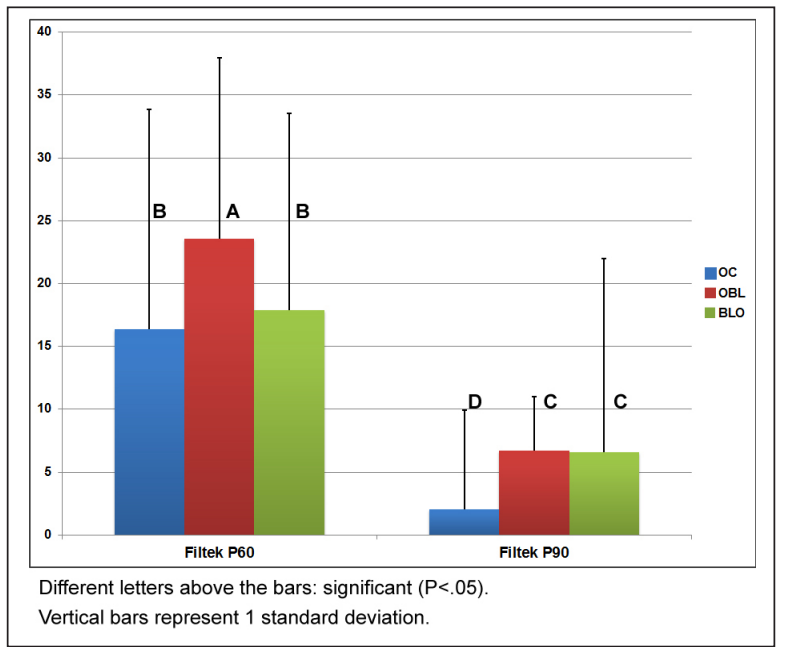

Figure 1. Internal gap percentage data. 2,000-grit SiC papers and submitted to internal gap and hardness evaluation.

Internal gap evaluation

After the samples were air dried, a drop of $1 \%$ acid red propylene glycol solution (Caries Detector, Kuraray, Osaka, Japan) was placed on the bonded interface for $20 \mathrm{~s} .{ }^{28}$ The samples were rinsed with water and air dried, and digital images were obtained. The length of staining along the interface was measured using Image Tool 2.0 software (UTHSC, San Antonio, TX, USA). Internal gap (\%) was calculated as the ratio of the stained interface to the total length of the interface. Data were submitted to nonparametric Kruskall-Wallis test at $5 \%$ significance.

\section{Hardness evaluation}

Knoop indentations were made across the section of the composite with an indenter (HMV-2, Shimadzu, Tokyo, Japan), using a $50 \mathrm{~g}$ load for $5 \mathrm{~s}$. Three readings were performed below the occlusal surface at 1, 2, 3, and $4 \mathrm{~mm}$ of depth. The Knoop hardness number (KHN) mean value was calculated from the three indentations for each depth. The data were submitted to three-way ANOVA and Tukey's test at a pre-set alpha of .05.

\section{RESULTS}

Internal gap evaluation

Internal gap percentage data can be seen in Figure 1. According to these results, silorane-based restorations showed significantly lower internal gaps than those in methacrylate-based restorations, regardless of photoactivation method employed $(P<.05)$. In addition, the two experimental photoactivation techniques produced different effects on internal adaptation of the restorations. For groups filled with the silorane-based composite, both OBL and BLO photoactivation methods produced more incidence of internal gaps in restorations than when only occlusal photoactivation was applied $(P<.05)$; for groups filled with the methacrylate-based composite, only $\mathrm{OBL}$ photoactivation technique produced a greater incidence of internal gaps in restorations than in conventional occlusally photoactivated restorations $(P<.05)$. BLO photoactivation technique showed similar results to those of the conventional occlusally photoactivated group for 3M ESPE Filtek P60 (P>.05). 


\section{Hardness evaluation}

Hardness results can be seen in Tables 2 and 3. ANOVA was conducted with three factors: composite $\mathrm{x}$ photoactivation $\mathrm{x}$ depth. For this test, statistical analysis showed that only the individual factors "composite" and "depth" and the interaction "composite $x$ photoactivation method" were significant $(P<$.05). The methacrylate-based composite Filtek P60 showed significantly higher mean Knoop hardness values than those of the silorane-based composite Filtek P90, regardless of other factors (Table 2). Regarding the factor of depth, no difference in KHN values was observed between 1 and $3 \mathrm{~mm}$ and between 2 and $4 \mathrm{~mm}$; however, both 1 and $3 \mathrm{~mm}$ below the restoration surface, both composites showed significantly higher hardness values than the values seen at both 2 and $4 \mathrm{~mm}(\mathrm{P}<.05)$. The $\mathrm{KHN}$ mean values can be ranked as follows: $1 \mathrm{~mm}=3 \mathrm{~mm}>2$ $\mathrm{mm}=4 \mathrm{~mm}$ (Table 2). The interaction between composite and photoactivation is shown in Table 3. For the methacrylate-based composite, the BLO group produced significantly higher hardness values compared with the values seen when the conventional occlusal photoactivation technique was applied $(P<.05)$; for the silorane-based composite no significant differences were observed among photoactivation methods. Triple interaction was not significant.

\section{DISCUSSION}

The first hypothesis, which anticipated that restorations filled with the silorane-based composite would present reduced gap formation, was accepted. Despite the polymerization technique, all groups in which Filtek P90 was applied presented significantly lower gaps than with Filtek P60. Various factors can be proposed to explain this difference. One of them relies on the different mechanisms of polymerization.

In methacrylate-based composites, a volumetric shrinkage occurs because of proximity of monomers that react to establish a covalent bond in the polymerization process. Besides, the distance between the two groups of atoms is reduced (from 4.0 to $1.5 \mathrm{~nm}$ ) and that also contributes to a reduction in free volume. ${ }^{29}$ Although this phenomenon also occurs with the silorane composite, the ring-opening chemistry promotes expansion of the molecule during the polymerization process. The kinetics of the initiation and polymerization begin with cleavage and opening of the ring systems via a cationic ring-opening reaction, allowing a gain of space that counteracts the reduction in free volume. ${ }^{10}$ Overall, the polymerization process yields reduced volumetric shrinkage; thus, less polymerization contraction $(<1 \%$ ) would create less polymerization stress compared with methacrylate-based composites (with volumetric contraction varying between 2 and $5 \%$ ); however, according to Marchesi et al, ${ }^{30}$ reducing shrinkage is not a guarantee of reduced stress development in composites. These authors found that shrinkage stress produced by silorane-based composites was comparable to that seen in methacrylate-based composites. Based on the results of the present study, it can be speculated that there might have been a reduction in shrinkage stress, as decreased gap formation was observed in silorane-based restorations.

Regarding photoactivation methods, both $\mathrm{OBL}$ and BLO techniques caused a significant increase in gap formation at the silorane composite-restoration interface. For the methacrylate-based composite, significantly higher gap formation was seen only when the $\mathrm{OBL}$ technique was applied after occlusal irradiation. In both cases, an increase in gap

Table 2. Means of hardness values according to composites, depth, and photoactivation techniques.

\begin{tabular}{ccccccccccccccc}
\hline & \multicolumn{1}{c}{ OC } & \multicolumn{1}{c}{ O B L } & \multicolumn{3}{c}{ B L 0 } \\
\hline & $1 \mathrm{~mm}$ & $2 \mathrm{~mm}$ & $3 \mathrm{~mm}$ & $4 \mathrm{~mm}$ & $1 \mathrm{~mm}$ & $2 \mathrm{~mm}$ & $3 \mathrm{~mm}$ & $4 \mathrm{~mm}$ & $1 \mathrm{~mm}$ & $2 \mathrm{~mm}$ & $3 \mathrm{~mm}$ & $4 \mathrm{~mm}$ \\
\hline \multirow{2}{*}{ Filtek P60 } & 85.1 & 80.1 & 82.2 & 78.3 & 86 & 83.1 & 83.6 & 82 & 87.3 & 82.6 & 88.2 & 83.2 \\
& 4.4 & 5.8 & 4.8 & 4.2 & 7.9 & 8.1 & 8.3 & 7.7 & 7.5 & 6.8 & 5.9 & 7.1 \\
\hline \multirow{2}{*}{ Filtek P90 } & 51.7 & 48.3 & 52.9 & 48.9 & 51 & 49.1 & 51 & 47.6 & 50.9 & 48.5 & 49.5 & 49.2 \\
& 2.9 & 3 & 4 & 5 & 4 & 3.5 & 2.7 & 2.9 & 2.8 & 4.6 & 3.1 & 3.9 \\
\hline
\end{tabular}

Table 3. Means of Knoop hardness (KHN) for interaction composite X photoactivation.

\begin{tabular}{|c|c|c|c|}
\hline & OC & OBL & BLO \\
\hline Filtek P 60 & $81.39 \mathrm{aB}$ & $83.65 \mathrm{aAB}$ & $85.30 \mathrm{aA}$ \\
\hline Filtek P 90 & 50.47 bA & $49.67 \mathrm{bA}$ & $49.51 \mathrm{bA}$ \\
\hline
\end{tabular}

Means followed by different small letters in column and capital letters in row: significant $(P<.05)$ 
formation can be explained by the energy dose applied to these composites. It is well known that the photoactivation method and the extent to which the polymerization reaction occur determine shrinkage values. ${ }^{29}$ According to Calheiros et al, ${ }^{31}$ an increase in energy dose after a certain limit causes an insignificant increase in degree of conversion but generates an intense and significant increase in shrinkage stress. In this way, the supplementary energy dose applied, especially in group $\mathrm{OBL}$, certainly caused an increase in gap formation at the internal margins in both composites. The energy dose somehow controls the degree of conversion, ${ }^{32}$ but the way this energy was applied (power density and type) to the composites also has an important effect, as confirmed by some studies. ${ }^{33,34}$ This energy-controlled conversion is particularly evident for the siloranebased composite. The higher the irradiation time, the greater the chances the oxirane rings will open, increasing the degree of conversion. But the extra energy might have raised the conversion in a rigid network in which the mobility of the developing polymer chains became progressively more restricted as a consequence of the increase in viscosity, reduction in free volume, formation of microgels and entanglement. ${ }^{35}$ Thus, increased conversion occurs after the gel point, at which time the developing polymer chains restrict the ability of the polymerizing network to flow in order to relieve the stress resulting from polymerization shrinkage. ${ }^{16,35}$ When the photoactivation method was only occlusally performed (OC group), the energy dose applied to these composites favored decreased shrinkage and probably reduced shrinkage stress. In this way, the second hypothesis, that the transdental polymerization technique would reduce gap formation without decreasing composite hardness, was rejected; however an interesting result occurred in the BLO group for methacrylatebased restorations. Despite the higher energy dose, which provided a higher degree of conversion (indirectly confirmed by the hardness test), gap formation seen in the BLO group was similar to that of the OC group. Thus, it can be speculated that increasing the irradiation period increased the energy dose applied to the composite, consequently increasing interfacial gaps, when compared with $20 \mathrm{~s}$ irradiation OOC group). However, in a comparison of BLO and OBL, irradiation directly on the resin composite (OBL) induced more gaps than transdental (BLO) irradiation.

Knoop hardness values were mainly influenced by the type of material. Hardness values seen with Filtek P60 were significantly greater than those for Filtek P90, regardless of photoactivation method $(P<.05)$. This can possibly be explained by the fact that the two composites are different in terms of filler concentration and type. Filtek P90 comprises a combination of fine quartz particles and radiopaque yttrium fluoride, classified as a microhybrid composite. The concentration of filler particles in this composite is $76 \%$ by weight. On the other hand, the filler in Filtek P60 is zirconia/silica in a concentration of $83 \%$ by weight. In contrast, the methacrylate-based composite is classified as a hybrid composite. For comparison, the $\mathrm{KHN}$ of quartz is 820 , while that of zirconia is $1,160 .{ }^{20}$ Previous studies have shown that variables such as size, shape, distribution, and content per volume/weight of filler particles in the matrix influence the material strength, hardness, and modulus of elasticity of resin composites. ${ }^{36,37,38}$

Regarding photoactivation methods, no significant difference was observed in the KHN of silorane-based composite groups, irrespective of photoactivation method used ( $P>$.05). For the methacrylate-based composite, significantly higher mean hardness values were observed for the BLO group when compared with the OC group $(\mathrm{P}<.05)$. This finding could be explained by the higher energy dose applied to the BLO group and possibly by the extended irradiation time, which allowed more free radical formation and more conversion. In a previous study, ${ }^{39}$ it was demonstrated that, considering the same composite, an increase in $\mathrm{KHN}$ was related to an increase in degree of conversion.

Initially, hardness across composite sections was determined to verify whether polymerization was adequate at all depths. It is well known that the number of photons reaching the bottom of the composite is reduced exponentially as thickness of the composite increases. ${ }^{40,41}$ In this way, the transdental photoactivation technique could improve polymerization at the bottom of the restorations because light, even at reduced irradiance, could reach the composite through the remaining buccal or lingual structure. However, such an improvement was not observed. Hardness values were not affected by photoactivation technique. All photoactivation provided adequate hardness values. Regarding the factor of depth, for both composites regardless of photoactivation technique, significantly higher hardness values were observed at 1 and $3 \mathrm{~mm}$ below the restoration surface than at 
2 and $4 \mathrm{~mm}$. Although significant hardness values were noticed, considering the factors of photoactivation method and depth, a difference less than 20\% in terms of this mechanical property would have no clinical significance. ${ }^{42}$ Consequently, considering the limit proposed by the manufacturer in terms of increment thickness $(2.5 \mathrm{~mm})$, it can be inferred by results of the hardness test that both composites were adequately polymerized throughout the material, irrespective of photoactivation method.

In a previous study ${ }^{43}$ it was found that a higher energy dose produces a slight increase in hardness for the silorane-based composite, but also increases the internal gap formation. On the other hand, it was point out that gap formation seems to be a consequence of an underperformed bonding approach rather than the differences in the resin-composite formulation. Extrapolations to clinically support and validate the results of the present study need to be done with caution since future studies are required.

\section{CONCLUSIONS}

Within the limitations imposed in this study, it can be concluded that

- Restorations filled with the silorane-based composite showed reduced gap formation compared to methacrylate, despite the polymerization protocol;

- A supplementary energy dose applied in experimental protocols causes an increase in internal gap formation for both composites;

- Silorane-based restorations exhibited lower hardness values but better internal adaptation compared with methacrylate-based restorations.

\section{REFERENCES}

1. Hilton TJ. Can modern restorative procedures and materials reliably seal cavities? In vitro investigations. Part 2. Am J Dent 2002;15:279-289.

2. Ferracane JL, Mitchem JC. Relationship between composite contraction stress and leakage in class v cavities. Am J Dent 2003;16:239-243.

3. Feilzer AJ, De Gee AJ, Davidson CL. Setting stress in composite resin in relation to configuration of the restoration. $J$ Dent Res 1987;66:1636-1639.

4. Braga RR, Ferracane JL. Alternatives in polymerization contraction stress management. Crit Rev Oral Biol Med 2004;15:176-184.

5. Versluis A, Douglas WH, Cross M, Sakaguchi RL. Does an incremental filling technique reduce polymerization shrinkage stresses? J Dent Res 1996;75:871-878.
6. Alonso RC, Cunha LG, Correr GM, De Goes MF, Correr-Sobrinho L, Puppin-Rontani RM, Sinhoreti MA. Association of photoactivation methods and low modulus liners on marginal adaptation of composite restorations. Acta Odontol Scand 2004;62:298-304.

7. Cunha LG, Alonso RC, Pfeifer CS, Correr-Sobrinho L, Ferracane JL, Sinhoreti MA. Modulated photoactivation methods: Influence on contraction stress, degree of conversion and push-out bond strength of composite restoratives. $J$ Dent 2007;35:318-324.

8. Amaral CM, Peris AR, Ambrosano GM, Pimenta LA. Microleakage and gap formation of resin composite restorations polymerized with different techniques. Am J Dent 2004; 17:156-160.

9. Ernst CP, Brand N, Frommator U, Rippin G, Willershausen B. Reduction of polymerization shrinkage stress and marginal microleakage using soft-start polymerization. J Esthet Restor Dent 2003;15:93-103.

10. Weinmann W, Thalacker C, Guggenberger R. Siloranes in dental composites. Dent Mater 2005;21:68-74.

11. Palin WM, Fleming GJ, Burke FJ, Marquis PM, Randall RC. The influence of short and medium-term water immersion on the hydrolytic stability of novel low-shrink dental composites. Dent Mater 2005;21:852-863.

12. Bagis $\mathrm{YH}$, Baltacioglu IH, Kahyaogullari S. Comparing microleakage and the layering methods of silorane-based resin composite in wide class ii mod cavities. Oper Dent 2009;34:578-585.

13. Schmidt M, Kirkevang LL, Horsted-Bindslev P, Poulsen S. Marginal adaptation of a low-shrinkage silorane-based composite: 1-year randomized clinical trial. Clin Oral Investig 2011;15:291-295.

14. Ak AT, Alpoz AR, Bayraktar O, Ertugrul F. Monomer release from resin based dental materials cured with led and halogen lights. Eur J Dent 2010;4:34-40.

15. D'Alpino PH, Svizero NR, Pereira JC, Rueggeberg FA, Carvalho RM, Pashley DH. Influence of light-curing sources on polymerization reaction kinetics of a restorative system. Am J Dent 2007;20:46-52.

16. Rueggeberg F. Contemporary issues in photocuring. Compend Contin Educ Dent 1999;25:S4-S15.

17. Halvorson RH, Erickson RL, Davidson CL. Energy dependent polymerization of resin-based composite. Dent Mater 2002; 18:463-469.

18. Kanca J, 3rd, Suh BI. Pulse activation: Reducing resinbased composite contraction stresses at the enamel cavosurface margins. Am J Dent 1999;12:107-112.

19. Yap $\mathrm{AU}, \mathrm{Ng}$ SC, Siow KS. Soft-start polymerization: Influence on effectiveness of cure and post-gel shrinkage. Oper Dent 2001;26:260-266. 
20. Belvedere PC. Controlling shrinkage using tep: Transenamel polymerization. Dent Today 1995;14:92,94-97.

21. Prati C, Chersoni S, Montebugnoli L, Montanari G. Effect of air, dentin and resin-based composite thickness on light intensity reduction. Am J Dent 1999;12:231-234.

22. Emami N, Soderholm KJ, Berglund LA. Effect of light power density variations on bulk curing properties of dental composites. J Dent 2003;31:189-196.

23. Asmussen E, Peutzfeldt A. Influence of selected components on crosslink density in polymer structures. Eur J Oral Sci 2001;109:282-285.

24. Rueggeberg FA, Caughman WF, Curtis JW, Jr. Effect of light intensity and exposure duration on cure of resin composite. Oper Dent 1994;19: 26-32.

25. Eliades GC, Vougiouklakis GJ, Caputo AA. Degree of double bond conversion in light-cured composites. Dent Mater 1987;3:19-25.

26. Harris JS, Jacobsen PH, O'Doherty DM. The effect of curing light intensity and test temperature on the dynamic mechanical properties of two polymer composites. J Oral Rehabil 1999;26:635-639.

27. Alves EB, Alonso RC, Correr GM, Correr AB, de Moraes RR, Sinhoreti MA, Correr-Sobrinho L. Transdental photoactivation technique: Hardness and marginal adaptation of composite restorations using different light sources. Oper Dent 2008;33:421-425.

28. Alonso RC, Cunha LG, Correr GM, Cunha Brandt W, Correr-Sobrinho L, Sinhoreti MA. Relationship between bond strength and marginal and internal adaptation of composite restorations photocured by different methods. Acta Odontol Scand 2006;64:306-313.

29. Braga RR, Ballester RY, Ferracane JL. Factors involved in the development of polymerization shrinkage stress in resin-composites: A systematic review. Dent Mater 2005;21:962-970.

30. Marchesi G, Breschi L, Antoniolli F, Di Lenarda R, Ferracane J, Cadenaro M. Contraction stress of low-shrinkage composite materials assessed with different testing systems. Dent Mater 2010;26:947-953.

31. Calheiros FC, Braga RR, Kawano Y, Ballester RY. Relationship between contraction stress and degree of conversion in restorative composites. Dent Mater 2004;20:939-946.

32. Calheiros FC, Daronch M, Rueggeberg FA, Braga RR. Degree of conversion and mechanical properties of a bisgma:Tegdma composite as a function of the applied radiant exposure. J Biomed Mater Res B Appl Biomater 2008;84:503-509.
33. Cunha LG, Sinhoreti MA, Consani S, Sobrinho LC. Effect of different photoactivation methods on the polymerization depth of a light-activated composite. Oper Dent 2003;28:155-159.

34. Uno S, Asmussen E. Marginal adaptation of a restorative resin polymerized at reduced rate. Scand $J$ Dent Res 1991;99:440-444.

35. Neves AD, Discacciati JA, Orefice RL, Yoshida MI. Influence of the power density on the kinetics of photopolymerization and properties of dental composites. J Biomed Mater Res B Appl Biomater 2005;72:393-400.

36. Peris AR, Mitsui FH, Amaral CM, Ambrosano GM, Pimenta LA. The effect of composite type on microhardness when using quartz-tungsten-halogen (qth) or led lights. Oper Dent 2005;30:649-654.

37. Oberholzer TG, Grobler SR, Pameijer $\mathrm{CH}$, Hudson AP. The effects of light intensity and method of exposure on the hardness of four light-cured dental restorative materials. Int Dent J 2003;53:211-215.

38. Manhart J, Kunzelmann KH, Chen HY, Hickel R. Mechanical properties and wear behavior of light-cured packable composite resins. Dent Mater 2000;16:33-40.

39. Bouschlicher MR, Rueggeberg FA, Wilson BM. Correlation of bottom-to-top surface microhardness and conversion ratios for a variety of resin composite compositions. Oper Dent 2004;29:698-704.

40. Price RB, Murphy DG, Derand T. Light energy transmission through cured resin composite and human dentin. Quintessence Int 2000;31:659-667.

41. Halvorson RH, Erickson RL, Davidson CL. An energy conversion relationship predictive of conversion profiles and depth of cure for resin-based composite. Oper Dent 2003;28:307-314.

42. Ferracane JL. Correlation between hardness and degree of conversion during the setting reaction of unfilled dental restorative resins. Dent Mater 1985;1:11-14.

43. D'Alpino PH, Bechtold J, dos Santos PJ, Alonso RC, Di Hipólito V, Silikas N, Rodrigues FP. Methacrylate- and silorane- based composite restorations: hardness, depth of cure and interfacial gap formation as a function of the energy dose. Dent Mater 2011;27:1162-1169. 